\title{
背景对齐差分的机场跑道异物分块检测与跟踪算法
}

\author{
王国屹，孙永荣，张怡，鲁海枰，赵伟 \\ (南京航空航天大学自动化学院导航研究中心 南京 211106) \\ (harrywang@nuaa.edu.cn)
}

\begin{abstract}
摘 要: 针对机场跑道异物的无人自主检测与识别过程中存在的检测结果受环境影响大、小目标检测困难以及漏检 率高等问题，提出基于背景差分的机场跑道异物分块检测与跟踪算法. 首先利用速度辅助的位置信息线性插值，获 取背景模板图像库与待检测图像序列最相关帧; 然后将图像分为不同子块, 利用各子块内图像纹理复杂度设置自适 应权值, 结合 ORB 算法进行特征点提取, 将各子块特征点归一化至原始图像, 并与背景模板库最相关帧对齐作差, 获取异物检测结果; 最后引人核相关滤波器对检测结果进行多目标跟踪, 采用各子块局部跟踪算法降低运算时间, 并对跟踪结果进行可靠性检验. 在 3 种实验场景下，与 5 种主流检测算法的对比实验结果表明，与目前已有的基于图 像的机场异物检测算法相比，在保证算法处理速度的基础上，该算法将异物整体错检率降低了 $70 \%$ 上，并在异物 尺寸大于 $1 \mathrm{~cm} \times 1 \mathrm{~cm}$ 的情况下, 将整体漏检率降低至 0 , 获得了较好的效果.
\end{abstract}

关键词：机场跑道异物；背景差分；自适应权值; 图像分块; 核相关滤波器; 目标检测跟踪

中图法分类号: TP391.41 DOI: 10.3724/SP.J.1089.2021.18387

\section{Block Detection and Tracking Algorithm of Foreign Objects Debris in Airport Runway Based on Background Alignment and Difference}

\author{
Wang Guoyi, Sun Yongrong, Zhang Yi, Lu Haiping, and Zhao Wei \\ (Navigation Research Center, College of Automation Engineering, Nanjing University of Aeronautics and Astronautics, Nanjing 211106)
}

\begin{abstract}
Aiming at the problems of unmanned detection and recognition of foreign object debris (FOD) in the autonomous detection and recognition of airport runways, the detection results are affected by the environment, the detection of small targets is difficult, and the rate of missed detection is high. An airport runway foreign object debris sub-block detection and tracking method based on background difference is proposed in this article. First, the linear interpolation of position information assisted by the speed is used to obtain the most relevant frame of the image in background image template library and the image sequence to be identified. Then, the image is divided into different sub-blocks, the adaptive weights are set using the image texture complexity in each sub-block, and the feature point extraction is combined with the oriented fast and rotated brief (ORB) method to normalize the sub-block feature points to the original image. Align the image with the most relevant frame of the background template library using the feature points result before to obtain the foreign object detection result. Finally, the kernelized correlation filter is introduced to track the detection results for multiple targets. Local tracking methods of each sub-block are used to reduce the computation time and the tracking results are tested for reliability. According to the experiment results in
\end{abstract}

收稿日期: 2020-05-13; 修回日期: 2021-01-04. 基金项目：国家自然科学基金(61973160). 王国屹(1996一), 男, 硕士研究生, 主 要研究方向为视觉导航、计算机图像处理; 孙永荣(1969-), 男, 博士, 教授, 博士生导师, 主要研究方向为惯性导航、视觉导航; 张怡(1997一), 女, 硕士研究生, 主要研究方向为视觉导航、计算机图像处理; 鲁海枰(1997一), 男, 硕士研究生, 主要研究方向为视 觉导航、目标检测; 赵伟(1971一), 男, 博士, 硕士生导师, 主要研究方向为组合导航、惯性传感器. 
three different scenarios, compared with another five common image-based airport FOD detection algorithms, this method can reduce the overall false detection rate of foreign objects by more than $70 \%$, and the overall missed detection rate to 0 when the size of foreign body is larger than $1 \mathrm{~cm} \times 1 \mathrm{~cm}$, while ensuring the algorithm processing speed, which achieved good results.

Key words: foreign object debris; background difference; adaptive weight; image blocking; kernelized correlation filter; object detection and tracking

机场跑道异物也称外来物(foreign object debris, FOD), 即有可能对航空器造成损伤的某种外 来物质、碎屑或物体 ${ }^{[1]}$, 其在对旅客生命安全造成 巨大威胁的同时，也给社会带来了难以估量的经 济损失. 传统 FOD 巡检方式主要依靠人工定时巡 视和人眼近距离搜寻，不但耗费时间长、效率低， 且对于较小异物的识别方法的可靠性较低. 因此, 世界多国针对性能和效率更高的 FOD 探测方法纷 纷展开研究 ${ }^{[2-4]}$.

针对跑道异物检测，国内外主要采用毫米波 雷达成像、红外热成像和光学成像 3 种技术. 针对 毫米波雷达, Nsengiyumva 等 ${ }^{[5]}$ 通过测量空间和偏 振分集的散射部分，利用毫米波波段对机场跑道 异物进行检测. 尽管应用较为广泛，毫米波雷达仍 存在制造成本和维护成本较高、目标特征信息较少 以及信号处理复杂等相关问题. 对于红外热成像 仪, 詹令明等 ${ }^{[6]}$ 利用目标红外特征研究了复杂背景 下的低信噪比红外弱小目标的检测方法. 然而红 外成像仍存在测距距离有限，硬件成本较高等问 题. 针对光学成像相机, 于之靖等 ${ }^{[7]}$ 提出了一种基 于小波变换与形态学的机场跑道异物检测算法. 但光学相机具有成像质量与视场范围的限制等问 题，导致其对于小目标检测、复杂环境下的目标检 测等存在一定不足.

对于利用光学成像的机场跑道异物检测系统 而言, 已有的方案主要采用移动式检测与固定式 检测 2 种方法 ${ }^{[8-9]}$. 移动式方法通过在跑道巡检车 上安装图像采集模块，利用快速目标检测方法如 ITTI 视觉显著性模型 ${ }^{[10]}$ 等进行处理, 但此种方法 对于小尺寸异物的检测效果不佳. 固定式方法通 过在跑道外区域设置的高杆上加装固定式图像采 集模块检测并识别异物，但由于相机放置在较高 位置且无法移动, 其单相机视场覆盖范围有限且 对于相机成像质量要求较高; 另外, 其安装位置所 需的高杆也对机场正常通航造成较大不便.

为解决上述 2 种方法中对机场跑道异物的无 人自主检测与识别过程中存在的检测结果受环境
影响大、小目标检测困难以及漏检率高的问题，本 文提出了一种利用背景差分与图像分块技术进行 准固定式机场跑道异物目标检测跟踪的算法.

\section{1 基于背景差分的权重自适应分块检测}

针对目前基于视觉图像的机场跑道异物检测 系统在跑道巡检图像覆盖范围、小尺寸目标的检测 效果以及对于机场通航要求等方面存在的局限性, 提出一种准固定式检测算法, 即基于背景差分的 移动式检测，利用特征点权重自适应分块检测算 法提高移动情况下小目标检测的准确性与鲁棒性.

\section{1 速度辅助的位置信息线性插值}

利用背景图像进行差分的首要任务是找到背 景模板图片库中与当前实时图像最相关的一帧. 由于跑道巡检轨迹具有单向性, 因此需引人位置 信息搜索最相关帧. 本文采用实时动态 (real-time kinematic, RTK)载波相位差分定位技术设备获取 图像采集过程的准确定位, 以此可以保证 2 组图像 采集起始位置与过程位置的偏差保持在较低(厘米 级)程度。

在背景模板图像库中, 搜索与当前图像最相 关帧需要依靠 RTK 信息匹配，但 RTK 的数据采集 时间间隔与图像采集帧间隔不存在匹配关系. 为 合理分配帧间 RTK 近似值, 考虑相机移动呈线性 变化特点, 需要采用线性插值的方法进行操作.

对于位置信息，传统的线性插值方法为

$$
y=\frac{x_{1}-x}{x_{1}-x_{0}} y_{0}+\frac{x-x_{0}}{x_{1}-x_{0}} y_{1}
$$

通过确定 2 个相邻 RTK 位置以及接收该位置 时的时间，计算 2 个位置之间的近似 RTK 信息. 以 RTK 信息中的经度 $X$ 为例，得到的计算结果为

$$
X_{i}=\frac{T_{j+1}^{\mathrm{R}}-T_{i}^{\mathrm{C}}}{T_{j+1}^{\mathrm{R}}-T_{j}^{\mathrm{R}}} X_{j}^{\mathrm{R}}+\frac{T_{i}^{\mathrm{C}}-T_{j}^{\mathrm{R}}}{T_{j+1}^{\mathrm{R}}-T_{j}^{\mathrm{R}}} X_{j+1}^{\mathrm{R}}
$$

将式(2)按照速度位置关系转化为 $X_{t}=v \cdot t+X_{0}$ 形 式, 可得 


$$
X_{i}=\frac{T_{i}^{\mathrm{C}}-T_{j}^{\mathrm{R}}}{T_{j+1}^{\mathrm{R}}-T_{j}^{\mathrm{R}}} \cdot\left(X_{j+1}^{\mathrm{R}}-X_{j}^{\mathrm{R}}\right)+X_{j}^{\mathrm{R}}
$$

其中, $T^{\mathrm{C}}$ 和 $T^{\mathrm{R}}$ 分别表示相机拍摄时间与 RTK 接 收时间; $i$ 和 $j$ 则分别表示图像帧序数与 RTK 信息 序数.

由于在接收到的 2 个相邻 RTK 位置之间运动 载体并非固定处于匀速运动情况，因此需引人对 速度变化的考量. 而考虑 2 个相邻 RTK 位置间由 于时间间隔较短，其加速度变化幅度较小，因此通 过加速度与位置的关系 $X_{t}=a \cdot t^{2} / 2+X_{0}$ 可以计算 每帧图像的近似 RTK 位置为

$X_{i}=\left[2 \cdot \frac{X_{j+1}^{\mathrm{R}}+X_{j-1}^{\mathrm{R}}-2 \cdot X_{j}^{\mathrm{R}}}{\left(T_{j+1}^{\mathrm{R}}+T_{j-1}^{\mathrm{R}}-2 \cdot T_{j}^{\mathrm{R}}\right)^{2}}\right] \cdot\left(T_{i}^{\mathrm{C}}-T_{j}^{\mathrm{R}}\right)^{2} / 2+X_{j}^{\mathrm{R}}$

如图 1 所示，以 1 周期内情况为例，每个周期 内可以固定获得 5 组 RTK 位置信息与 19 帧相机回 传图像，因此需将这 5 组 RTK 位置信息通过线性 插值得到全部 19 帧图像的近似 RTK 位置信息, 再 对比背景库内各图像与待检图像的近似 RTK 结果, 求得背景库的最相关帧。

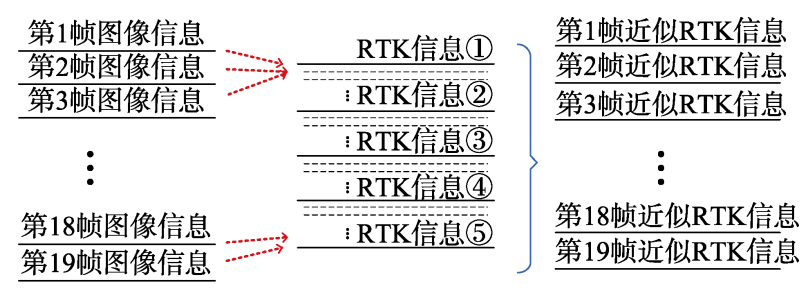

图 1 位置信息线性插值示意图

\section{$1.2 \mathrm{ORB}$ 特征点自适应加权提取的分块检测}

最相关帧的获取使得背景差分所需的 2 幅图 像得以确定，但由于载具运动过程或相机拍摄角 度存在偏差, 以及由 RTK 获取的位置信息本身存 在一定误差，以上问题均会有损图像直接差分的 效果, 因此需要利用目标与背景特征进行图像对 齐操作。本文采用旋转不变性特征提取 (oriented fast and rotated brief, ORB)算法提取目标与背景特 征点. 然而机场跑道环境由于飞行管控等因素, 其 周围大多数情况下较为空旷, 这使得图像中的画 面信息较少，不利于 ORB 算法提取特征. 因此, 利用图像分块处理方法可以突出待选特征点区域 细节信息，有效地解决特征点少的问题. 利用分块 结果自适应计算各子块内 ORB 特征点提取数目的 权重, 并对特征点进行加权提取. 将特征点归一化 至图像全局进行图像对齐作差，利用差分结果检
测跑道异物.

\subsection{1 图像叠式分块}

通常机场巡检车上搭载的相机视角下的机场 跑道场景包含近处跑道与远处背景 2 部分, 跑道区 域图像信息较少，而远处背景区域图像信息相对 较多. 由于 ORB 算法中为保证运算速度, 特征点 的选取具有数量限制, 因此对于机场跑道区域, 图 像远处背景部分提取的特征点数量会抑制跑道部 分提取的特征点数量, 导致图像对齐时出现局部 最优问题，增大了图像对齐误差，故而图像分块时 应尽量将跑道与背景分离. 根据常规跑道巡检车 辆运行状态下相机的拍摄视角通常为沿车辆行驶 方向并向下一定角度, 在图 2 中可见在这种视角下 画面内跑道区域呈上窄下宽的梯形区域, 背景区 域多集中在图像的上半部分且位于梯形区域外, 因此将图像分为上下 2 部分, 可初步分离跑道与背 景. 同时由于跑道在图像中成像为梯形这一特点, 在图像上半部分内仍存在一些跑道区域, 因此将 图像分为左中右 3 列, 可以更好地区分目标跑道区 域与背景区域. 综上, 本文将图像分为尺寸相等的 2 行 3 列共 6 子块, 如图 2 所示.

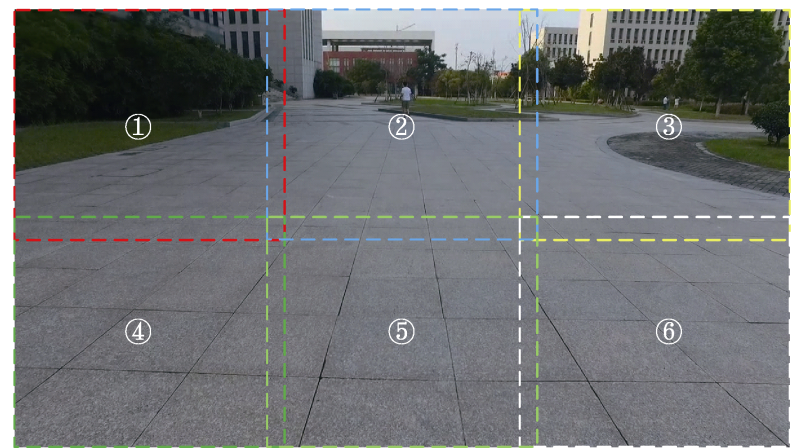

图 2 叠式分块示意图

将图像分块后由于小目标有可能出现在子块 衔接区域, 导致检测结果出错或出现遗漏, 因此在 分块时对各子块采用叠式分割方法, 即对各子块 设计补丁区域, 以此解决子块衔接区域的小目标 检测结果易出错问题。各子块补丁区域重合部分 长度的设置如图 2 所示, (1)(3)(4)(6)子块中每个子块 对应 2 个补丁区域, 每个补丁长度均为 20 像素; (2)(5) 2 个子块均对应 3 个补丁区域, 其中与左右相 邻子块相接的补丁区域的左右补丁长度均为 10 像 素，而该 2 个子块相接的补丁区域长度为 20 像素. 以此来保证分割后的各子块尺寸相同, 便于对各 子块内提取的异物位置进行全图归一化计算，获 取其在全图内的坐标. 同时, 选取 10 像素与 20 像 
素距离作为不同子块的不同补丁区域长度, 也是 基于对补丁区域内异物检测效果与补丁区域带来 的算法额外复杂度的综合平衡考量, 并根据实际 情况反复试验得到的较优解.

\subsubsection{ORB 特征点提取过程的自适应权重计算}

对各子块灰度化处理后分别计算块内像素灰 度值的标准差 $\sigma$ (即均方差), 其可以反映图像的信 息复杂度; $\sigma$ 越大, 表示图像信息越复杂, 即可提 取的特征点越多. 利用各子块标准差 $\sigma_{i}$ 与各子块 标准差中的最小值 $\sigma_{\min }$ 作比较, 利用归一化思想 求得各子块权重

$$
\omega_{i}=\mathrm{e}^{\lg \left(\frac{\sigma_{i}}{\sigma_{\min }}\right)}
$$

利用 ORB 算法将子块内检测到的全部 ORB 特征点按可靠性程度由高到低排列得到 $S_{i}^{j}$, 并根 据加权后拟提取数目获得的特征点即为各子块加 权提取特征点的结果

$$
S_{i}=S_{i}^{j} \times \omega_{i}
$$

由各子块得到的特征点总数目 $S$, 并将所有 子块特征点所在位置归一化至原图坐标, 即

$$
\left\{\begin{array}{l}
x_{j}^{i *}=x_{j}^{i}+x_{0} \\
y_{j}^{i *}=y_{j}^{i}+y_{0}
\end{array}\right.
$$

其中, $x^{*}, y^{*}$ 和 $x, y$ 分别表示各子块特征点与归一 化至全图后特征点的横纵坐标; $i$ 和 $j$ 分别表示子 块序号与该子块内特征点序号; $x_{0}, y_{0}$ 则表示各子 块左上角坐标. 经加权操作后提取到的各子块 ORB 特征点如图 3 所示.

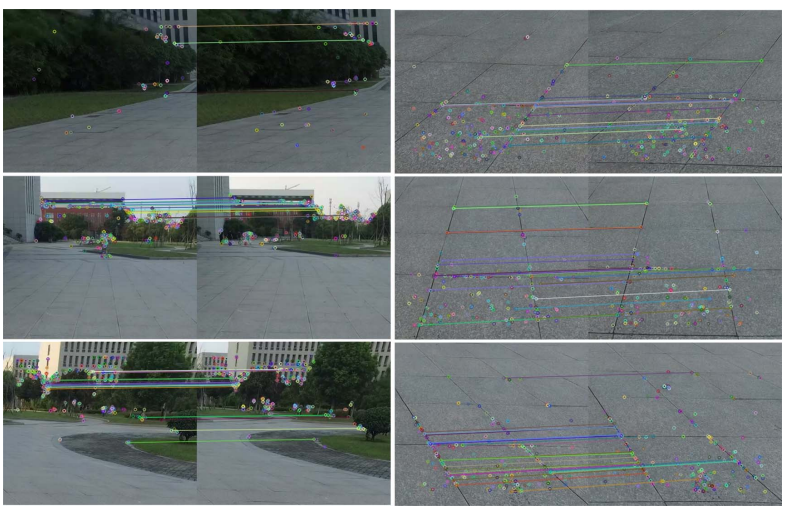

图 3 各子块 ORB 特征点加权提取结果图

\subsection{3 图像对齐差分与异物检测}

得到图像全局特征点，利用仿射变换将当前 帧图像对齐至背景库最相关帧图像, 并将对齐结 果与之进行差分运算. 对差分图中结果进行图像
处理后, 提取异物边缘点得到异物检测结果, 并绘 出其外接矩形作为检测结果显示, 其过程以及图 像对齐、差分与异物检测结果(以第 206 帧为例)如 图 4 和图 5 所示. 其中图 5e 中黄色框为检测结果, 场景中最小的异物尺寸为 $1 \mathrm{~cm} \times 1 \mathrm{~cm}$. 该过程的 2 步灰度阈值处理步骤中, 阈值的选取均要保证在 保留异物信息的同时, 尽可能地篎除无用噪声信 息. 因此通过多次试验验证, 将第 1 次灰度阈值操 作的阈值选择为 30 , 第 2 次的选择为 40 , 这 2 组國 值参数的选择均为工程经验的结果.

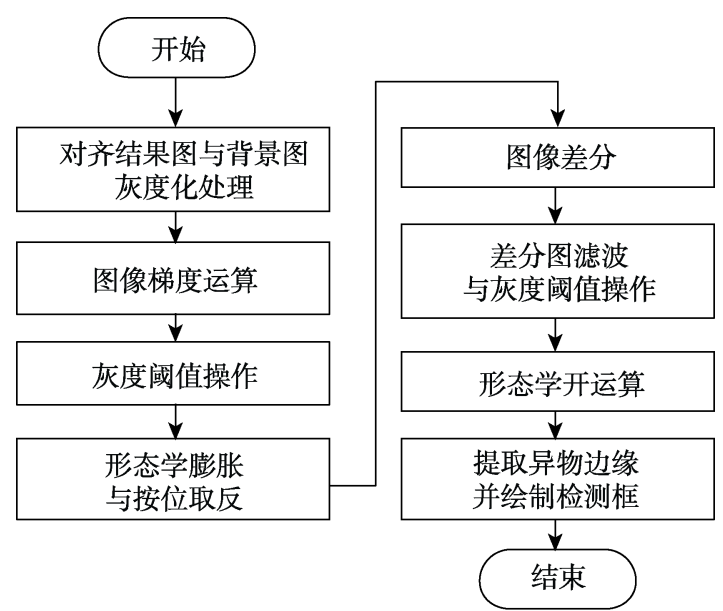

图 4 图像对齐差分与异物检测流程图

\section{2 基于核相关滤波器的多目标局部跟踪}

利用背景差分进行机场跑道异物检测的方法 可以有效地提高对异物, 尤其是对小目标的检测 成功率, 并降低错检率与漏检率. 但由于相机载具 运动过程中仍有可能出现突然抖动导致图像对齐 精度降低, 或者其他特殊情况造成异物漏检和错 检等问题, 此时检测结果不再准确, 无法用于确定 异物位置. 因此需引入针对检测结果的目标跟踪 方法, 采用跟踪结果定位异物. 传统单目标跟踪方 法如 MeanShift ${ }^{[11]}$ 等对于小目标, 尤其是运动速度 较快的目标跟踪效果不佳; 多目标跟踪方法如 $\mathrm{SORT}^{[12]}$ 等存在相似目标标签切换次数过于频繁的 问题; 基于神经网络的跟踪方法如 Deep-SORT ${ }^{[13]}$ 等, 则需要搭建包含大量数据的样本库进行预训 练方可用于实时跟踪, 其工作量较大. 因此, 为进 一步降低错检率和漏检率，多目标跟踪方法用核 相关滤波器 ${ }^{[14]}$ (kernelized correlation filter, KCF)对 多个检测结果进行多目标跟踪, 并根据第 1 节中图 像分块结果在子块区域内采取局部跟踪方法, 以 此提升系统处理速度，保证整体算法运行时间. 


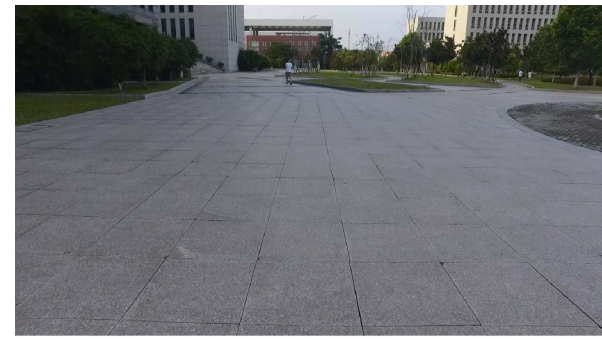

a. 第206帧对应背景图(对应背景库中第189帧)

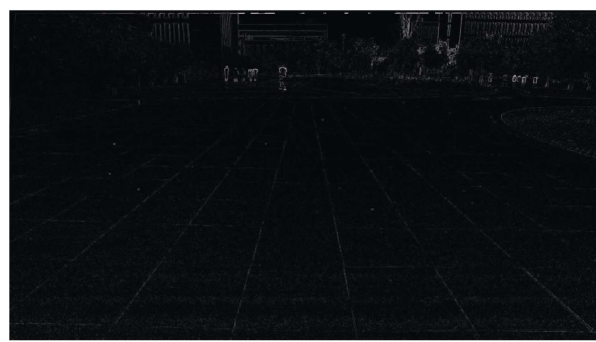

c. 第206帧对齐结果差分图

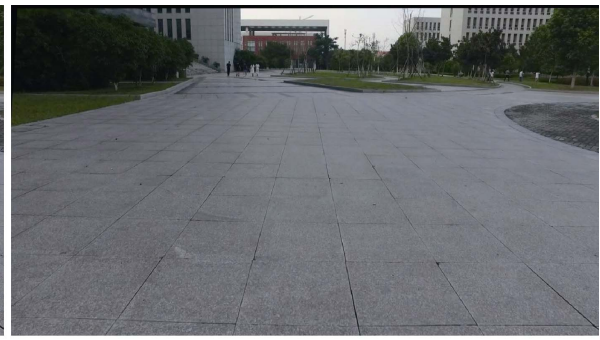

b. 第206帧对齐结果图

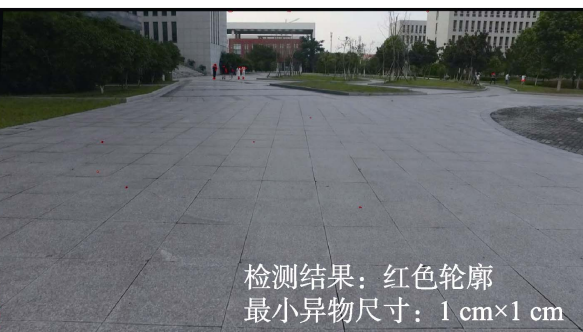

d. 第206帧异物检测边缘图

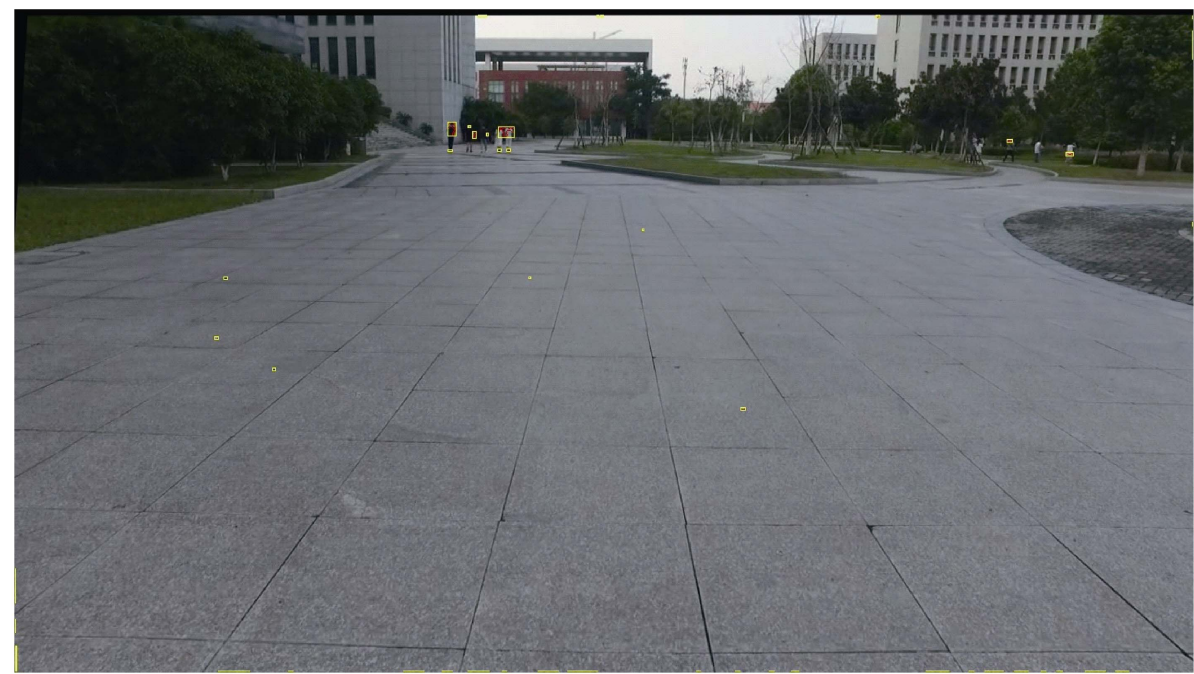

e. 第206帧检测结果图

图 5 图像对齐差分与异物检测结果相关示意图

\section{1 多目标下 KCF 的初始化}

为实现实时跟踪, KCF 已开始被广泛应用 ${ }^{[15]}$. KCF 跟踪算法是一种判别式核相关跟踪方法，采 用脊回归的方式训练检测器, 并利用循环矩阵将 矩阵的运算转化为矩阵内部元素的点积, 在保证 跟踪结果准确性的条件下，大大提高了运算速度， 使其可以达到 150 帧/s 以上 ${ }^{[16]}$. 但 KCF 方法主要 面向单目标跟踪 ${ }^{[14]}$, 为利用该方法实现多目标跟 踪过程, 本文采用基于检测结果初始化多跟踪器 分别跟踪的方法. 首先需检验当前检测结果与已 有跟踪目标的相关性, 并引人余弦相似度进行判 断. 余弦相似度 $\rho_{X, Y}$ 又称余弦相似性或余弦距离, 可以用于测量 2 个目标在向量空间中的向量夹角 余弦值, 以衡量 2 个目标之间的相关性程度, 计算 方法为

$$
\rho_{X, Y}=\frac{X \cdot Y}{\|X\|\|Y\|}=\frac{\sum_{i=1}^{n} X_{i} \times Y_{i}}{\sqrt{\sum_{i=1}^{n}\left(X_{i}\right)^{2}} \times \sqrt{\sum_{i=1}^{n}\left(Y_{i}\right)^{2}}}
$$

由于计算相似度时目标向量采用各目标颜色 直方图信息, 因此其中 $X, Y$ 分别表示 2 个目标框 内的颜色直方图向量信息; $n$ 表示颜色直方图颜 色值个数. 该计算结果取值区间为 $[-1,1]$, 若其绝 对值越接近 1 , 则表示目标相关性越强; 绝对值越 小, 则表示目标相关性越弱.

利用基于颜色直方图的余弦相似度 $\rho_{X, Y}$ 对当 前帧异物检测结果与已有跟踪器目标进行相关性 检验, 设置相关性阈值 $\phi$ 用于判断各检测结果是 否与已有跟踪器目标一致, 即 


$$
\left\{\begin{array}{l}
\rho_{X, Y}>\phi, \text { 目标一致 } \\
\rho_{X, Y}<\phi, \text { 目标不同 }
\end{array}\right.
$$

若一致, 则跳过该目标检测结果; 否则为该检测结 果创建新的跟踪器. 在跟踪过程中, 由于跟踪框可 能存在一定的漂移或尺度不敏感情况，因此根据 多次试验得到的经验, 此处的相关性阈值的取值 设置为 $\phi=0.7$. 该多目标跟踪器初始化过程具体 操作流程如图 6 所示.

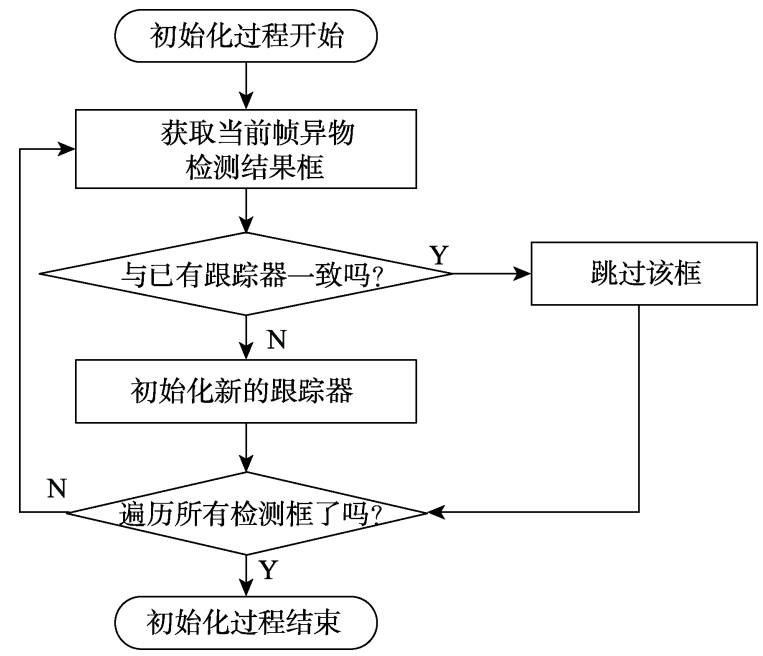

图 6 KCF 多目标跟踪器初始化流程图

\section{2 多子块局部跟踪}

为每帧检测结果引入多个跟踪器, 本质上解 决了基于 KCF 方法的多目标跟踪问题，但每出现
新的检测结果均将初始化新的跟踪器, 且原始 KCF 方法设定采用全局跟踪模式 ${ }^{[14]}$, 导致跟踪器 数量出现累积且各跟踪器均以全图信息进行位置 预测更新, 进而影响算法处理速度. 为解决多目标 跟踪的快速性问题, 采用局部跟踪的方法降低每 帧图像跟踪区域的尺寸，将子块区域图像信息用 于跟踪器位置结果更新, 进而降低因跟踪器数目 累积对运算速度造成的影响.

如图 7 所示, 以单目标为例, 根据检测结果初 始化跟踪器时定位目标在图像内的位置, 确定所 属子块, 并将该子块的图像作为该跟踪器的跟踪 区域，下一帧时同样在本区域内进行跟踪结果的 更新. 根据跟踪框的更新结果判断此时跟踪框坐 标是否在该子块区域范围内, 即跟踪框 4 个顶点的 坐标是否已移出该子块区域. 若已移出本子块区 域, 则继续判断跟踪框是否仍在整个图像区域范 围内. 若目标跟踪框已移出图像区域, 则设置该跟 踪器跟踪任务结束并释放; 否则根据跟踪框最后 位置、结合图像叠式分块结果确定目标即将出现的 区域所属子块, 并在新的子块中重新初始化跟踪 器继续跟踪, 具体操作流程如图 8 所示.

由于 KCF 方法本身存在尺度不敏感性 ${ }^{[14]}$, 因 此当图中跟踪结果尺度变化较大时易出现跟踪偏 移情况, 导致跟踪目标错误、跟踪失败. 故通过设 置跟踪结果可靠性检验, 来判断跟踪器的跟踪是 否成功与跟踪过程是否应继续.

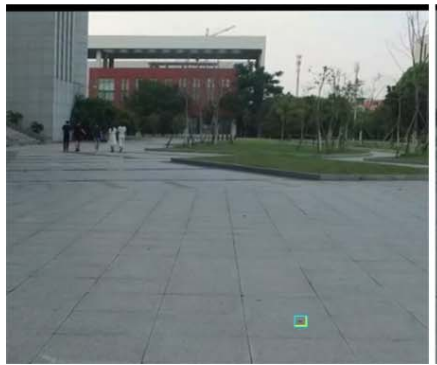

a. 子块(2)跟踪开始

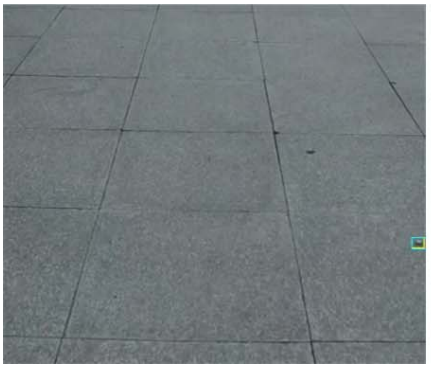

d. 目标出子块(4)

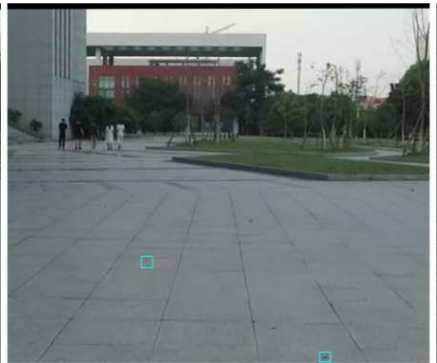

b. 目标出子块(2)

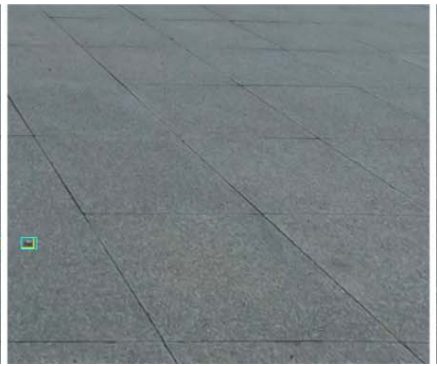

e. 目标人子块 (5)

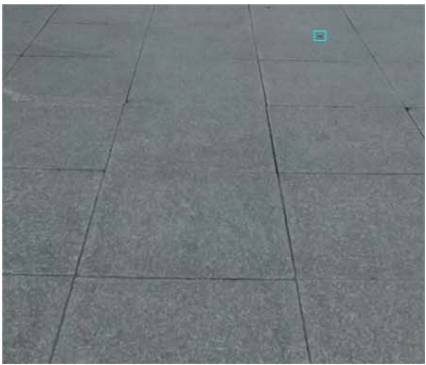

c. 目标人子块(4)

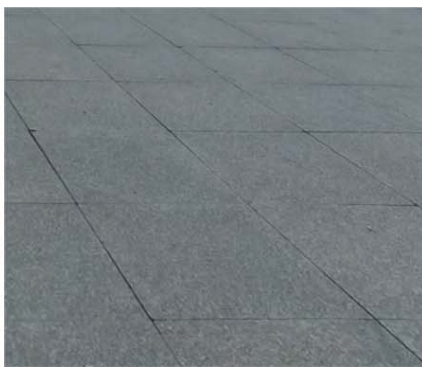

f. 目标出图

图 7 单目标多子块局部跟踪示意图 


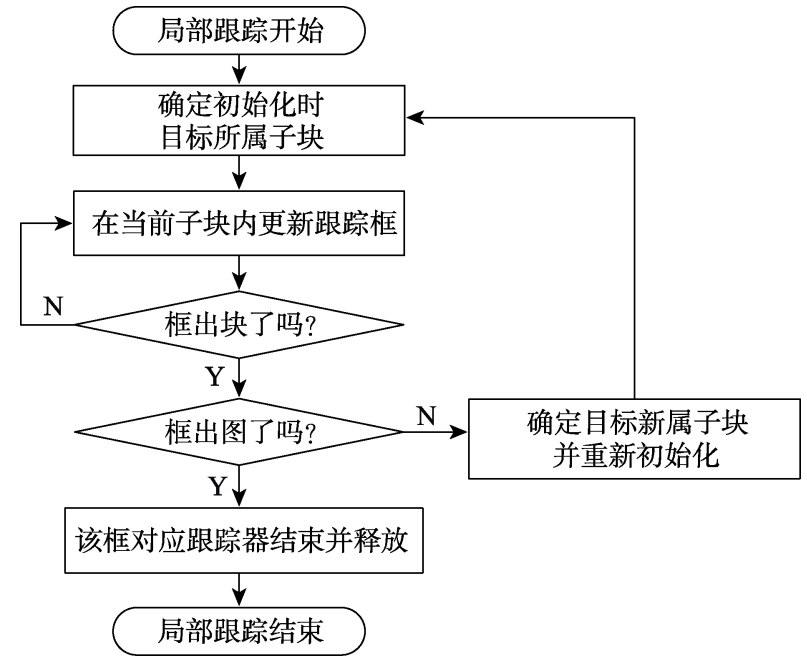

图 8 局部跟踪流程图

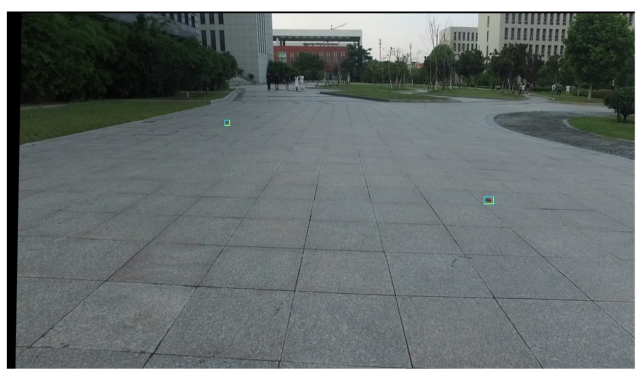

a. 第 1 帧跟踪初始化

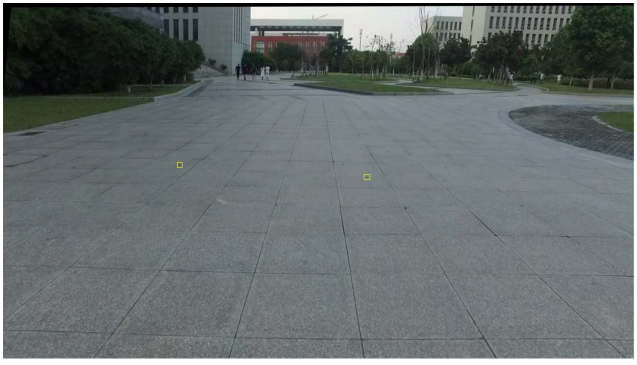

c. 第 169 帧纯检测结果
通过计算当前跟踪结果与初始跟踪目标图像 的余弦相似度, 并判断其是否漂移过大造成跟踪 目标错误. 若计算结果小于预设阈值 $\hat{\phi}$, 则判断该 跟踪器跟踪任务结束并将其释放. 判断方法为

$$
\left\{\begin{array}{l}
\rho_{X, Y}>\hat{\phi}, \text { 跟踪正常 } \\
\rho_{X, Y}<\hat{\phi}, \text { 跟踪错误 }
\end{array}\right.
$$

由于跟踪结果的判断影响到其异物的检出情 况，需严格判断跟踪任务是否可以正常进行，因 此将此处的跟踪结果判断阈值的取值设置为 $\hat{\phi}=0.9$.

为测试多目标的多子块局部跟踪算法可行性, 以某一场景实验为例, 经过跟踪算法得到的异物 跟踪效果如图 9 所示.

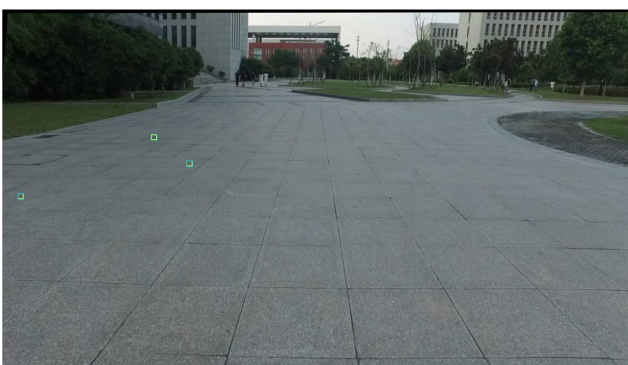

b. 第 153 帧新目标初始化

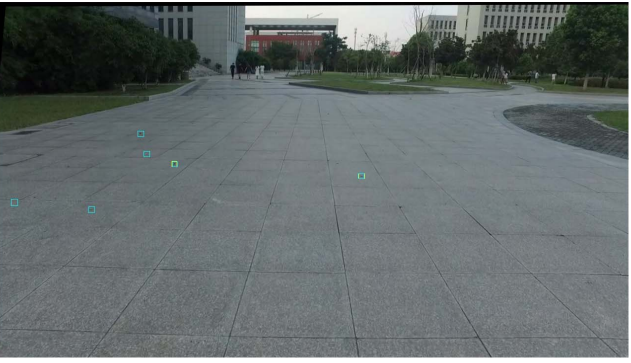

d. 第 169 帧检测+跟踪结果

图 9 跟踪算法效果图

引人跟踪算法后，在每帧的检测结果框周围设 定搜索区域寻找区域内跟踪结果框, 并对 2 个框进 行 IoU 阈值篮选. 若大于预设阈值，表明该异物检测 与跟踪结果一致，则输出检测框位置判断异物位置; 若小于预设阈值，表明该异物检测结果存在可疑性， 则输出跟踪框位置判断异物位置; 若区域内无跟踪 框，则认为该检测结果为新出现异物，此时输出检 测框位置用于计算异物位置. 由于需保证判断可靠 性，因此根据多次试验经验，此处阈值设为 0.9 .

\section{3 实验与结果讨论}

为验证本文算法有效性，以多段校园道路模拟 机场跑道环境场景，在不同场景下采集多组未放置
异物的背景图像序列与放置异物的待检测图像序列 进行算法验证，通过计算多场景下算法的异物错 检率和漏检率，并与简单背景差分法 ${ }^{[17]}$ 、动态 ITTI 检测法 ${ }^{[18]}$, Canny 边缘检测法 ${ }^{[19]}$ 、数学形态学检测 法 $^{[20]}$ 以及区域生长检测法 ${ }^{[21]}$ 进行结果对比分析. 本实验采用 Novatel OEM-615 RTK 移动站接收机与 大疆禅思 X3 相机云台采集数据, 算法运行的软件环 境为 Windows 10 系统下的 Visual Studio 2012, 采用 $\mathrm{C}++$ 作为编程语言; 硬件环境为 Intel Core i9-9800H, 内存 $16 \mathrm{~GB} .3$ 组实验场景分别采用石砖地面与柏油 地面, 模拟正常滑行跑道区域、起降(有刹车印)跑道 区域以及停机坪区域，场景示意如图 10 所示. 实验 中跑道异物采用石子、螺钉、螺帽、铁块等物品进 行模拟, 如图 11 所示, 异物尺寸范围在 1 8 cm. 


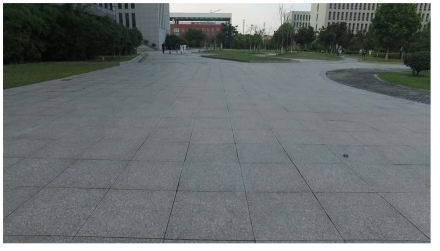

a. 场景 1

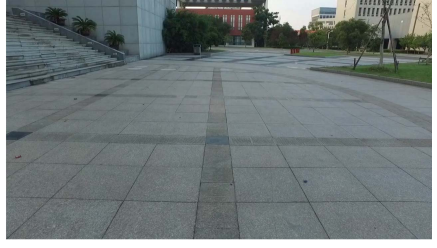

b. 场景 2

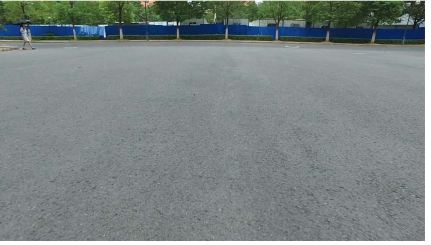

c. 场景 3

图 103 组实验场景示意图

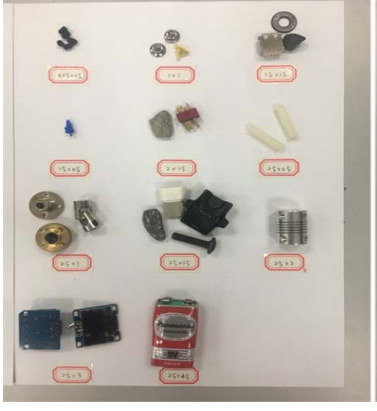

a. 小异物样本

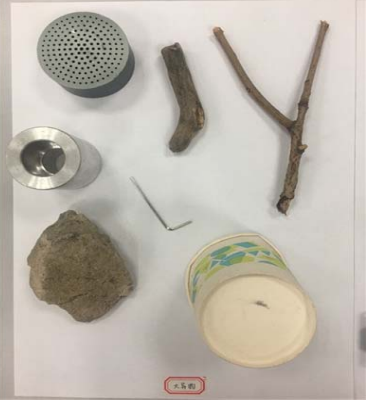

b. 大异物样本
图 11 模拟异物样本示意图

\section{1 异物错检率实验结果与分析}

错检率指经算法检出但实际并非异物的目标 数量与算法检测结果所认为是异物的全部目标数 量之比, 可以体现算法对于图像对齐差分结果的 误差程度, 表示算法对于异物检出结果的准确性 以及对于异物检出准确程度的鲁棒性.
图 12、图 13 分别为 3 组实验场景下 6 种算法 的错检数目与错检率对比图, 其中横坐标为视频 帧的帧序号, 纵坐标分别为错检数目与错检率. 在 3 组实验中, 简单背景差分检测由于其对齐效果的 限制, 异物检测的错检率较高, 易将背景环境判别 为异物导致检测错误; 动态 ITTI 检测算法的错检 数目较少, 但错检率要明显高于本文算法, 这是由 于其对于小目标异物检测效果较差, 导致每帧整 体图像内所检测到的异物较少, 主要为大目标异 物, 且易受环境内背景信息影响; 另外 3 种算法由 于检出结果中存在大量错误目标, 因此其错检数 目和错检率均高于本文算法. 在场景 3 中, 由于实 验环境采用柏油路模拟跑道, 而柏油路面颗粒感 较强、质地粗糙，因此导致 6 种算法的错检程度都 较高. 表 1 所示为 6 种算法在 3 组实验场景下整体 错检率情况.

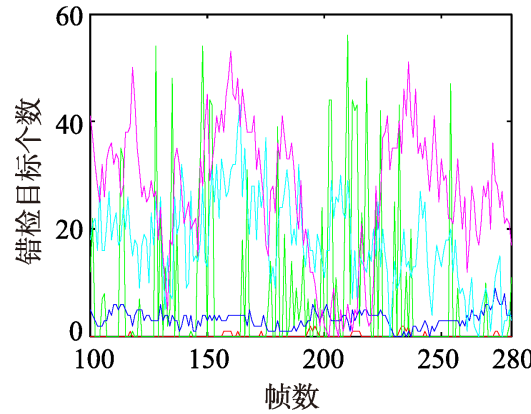

a. 场景1

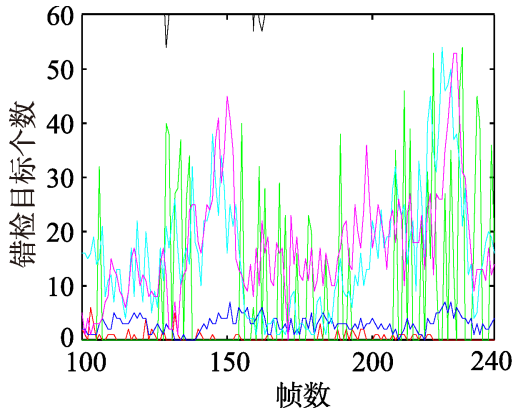

b. 场景 2

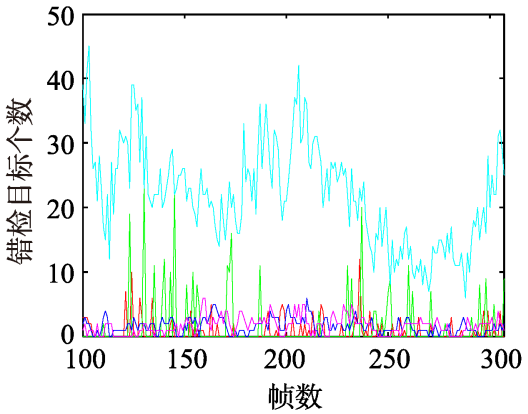

c. 场景 3

一 本文; 一一 简单背景差分检测 [17]; 一 动态ITTI检测 ${ }^{[18]} ;$ - Canny边缘检测 ${ }^{[19]} ;$ 一 数学形态学滤波 ${ }^{[20]} ;$ 一 区域生长检测 ${ }^{[21]}$

图 12 不同场景下 6 种算法错检数目对比图

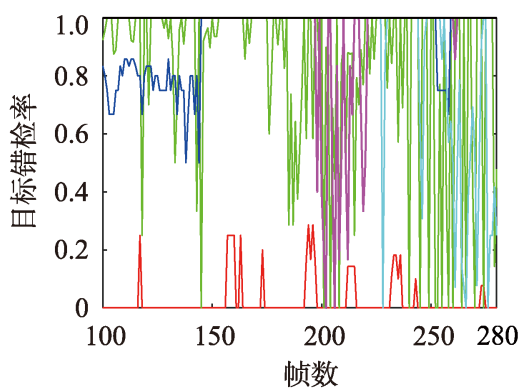

a. 场景1

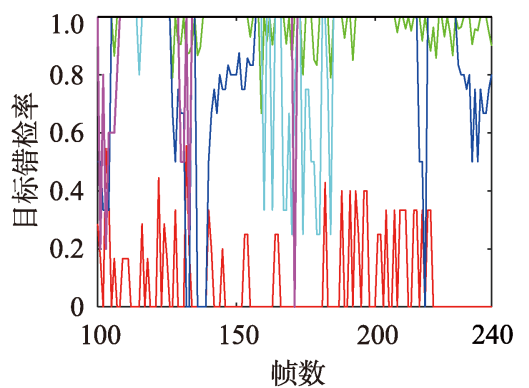

b. 场景 2

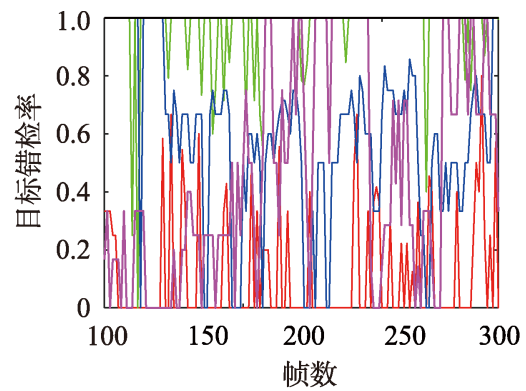

c. 场景3

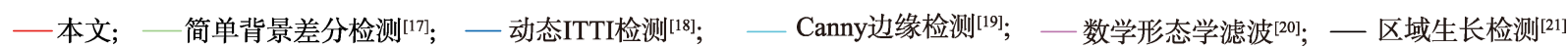

图 13 不同场景下 6 种算法错检率对比图 
表 1 不同算法整体错检率对比

\begin{tabular}{lrrrr}
\hline \multicolumn{1}{c}{ 算法 } & 场景 1 & 场景 2 & 场景 3 & 平均 \\
\hline 本文 & 2.41 & 8.90 & 11.77 & 7.69 \\
简单背景差分 $^{[17]}$ & 81.57 & 97.09 & 94.32 & 90.99 \\
动态 ITTI 检测 $^{[18]}$ & 93.29 & 85.76 & 56.74 & 78.59 \\
Canny 边缘检测 ${ }^{[19]}$ & 91.42 & 93.74 & 100.00 & 95.05 \\
数学形态学滤波 $^{[20]}$ & 94.71 & 95.84 & 53.16 & 81.24 \\
区域生长检测 ${ }^{[21]}$ & 100.00 & 100.00 & 100.00 & 100.00 \\
\hline
\end{tabular}

综合比较 6 种算法，本文算法每帧错检程度最 轻, 整体错检率最低, 查准效果最好.

\section{2 异物漏检率实验结果与分析}

漏检率指本应被检出为异物但未检出的目标 数量与待检全部异物数量的比值, 其可以体现算 法对于异物的敏感程度，表示算法对于异物检出 数量的完整性.

图 14、图 15 分别为 3 组实验场景下 6 种算法 的漏检数目与漏检率对比图, 其中横坐标为视频 帧的帧序号, 纵坐标分别为漏检数目与漏检率. 在 3 组实验中，动态 ITTI 检测法由于对于小目标的不 敏感性, 因此其漏检数目较多、漏检率高; 简单背 景差分法理论上漏检数目与漏检率应较低, 但由
于对齐效果的限制, 当图像对齐精度较高时其漏 检方面表现较好, 而图像对齐精度较低时其漏检 方面表现较差, 因此在图像中呈现忽高忽低的变 化趋势; 另外 3 种算法由于其检出结果数目较多, 因此对于待检目标的包含程度较高, 漏检率较低, 但仍会出现一定的漏检情况. 表 2 所示为 6 种算法 在 3 组实验场景下整体漏检率情况.

综合比较 6 种算法，本文算法每帧漏检程度最 轻, 如表 2 所示, 在实验中采用不小于 $1 \mathrm{~cm} \times 1 \mathrm{~cm}$ 尺寸的异物情况下, 其整体漏检率最低, 查全效果 最好; 即使在某些帧内出现漏检情况, 但对于整个 图像采集的相机运动过程中可以做到对于地面所 有异物的百分之百检测.

总体而言，本文算法在漏检与错检情况中比 其他 5 种算法具有较大的提升, 但仍存在一定不足 之处. 首先, 由于在检测过程中引人权重自适应分 块以及局部跟踪算法，因此在算法处理速度方面 处理速度为 1.429 帧/s, 表现不如简单背景差分法 和动态 ITTI 检测法. 其次, 由于实验条件所限, 本 实验仅在 3 个校园场景下进行测试验证, 未能在真 实的机场跑道场景进行, 因此在实际跑道中的算

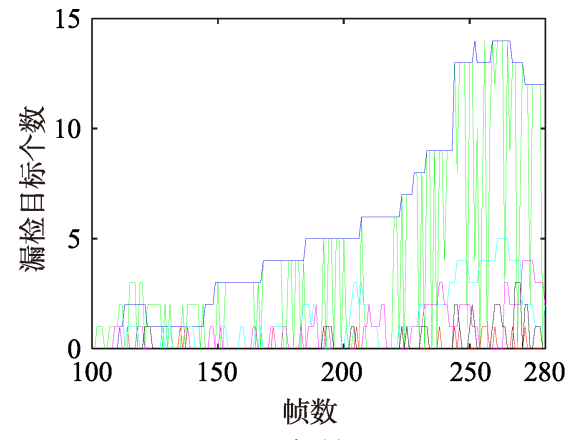

a. 场景1

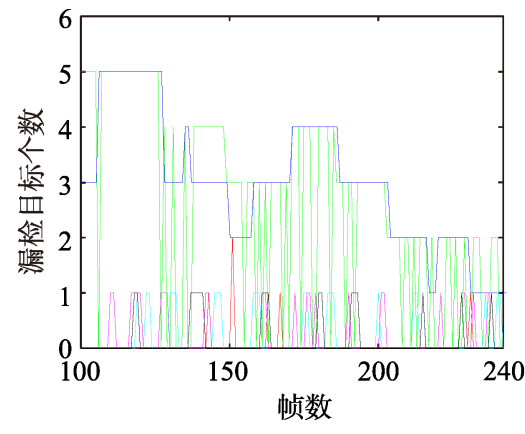

b. 场景 2

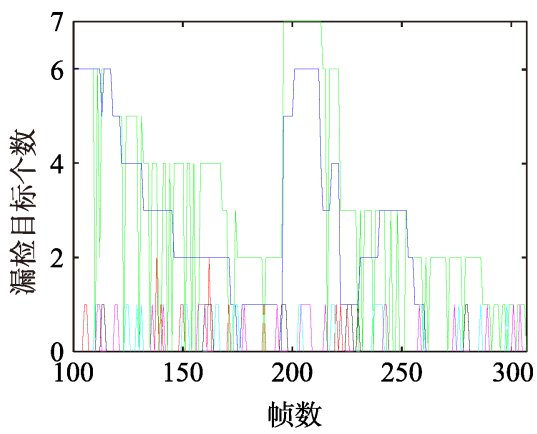

c. 场景3

一本文; 一 简单背景差分检测 [17]; — 动态ITTI检测 ${ }^{[18]}$;

- Canny边缘检测 ${ }^{[19]} ;$ — 数学形态学滤波 ${ }^{[20]} ;$ — 区域生长检测 ${ }^{[21]}$

图 14 不同场景下 6 种算法漏检数目对比图

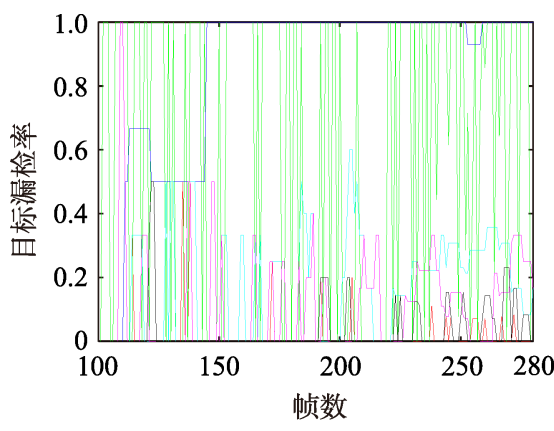

a. 场景1

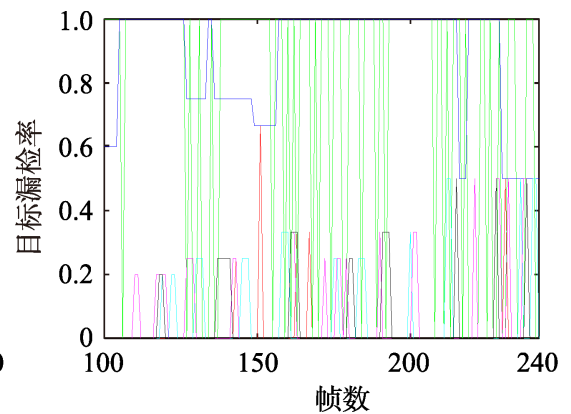

b. 场景2

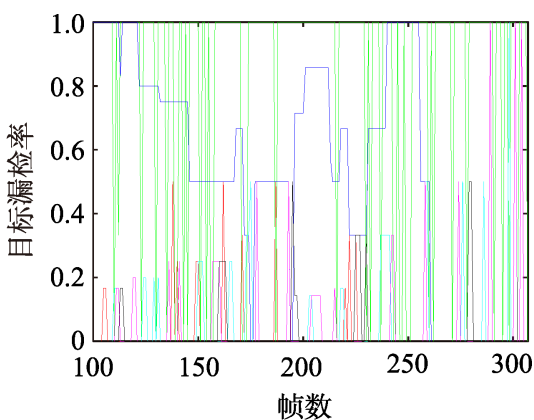

c. 场景3

一 本文; 一 简单背景差分检测 [17]; 一 动态ITTI检测 ${ }^{[18]}$;

— Canny边缘检测 ${ }^{[19]}$; — - 数学形态学滤波 ${ }^{[20]}$; 一 区域生长检测 ${ }^{[21]}$

图 15 不同场景下 6 种算法漏检率对比图 
表 2 不同算法整体漏检率对比

\begin{tabular}{|c|c|c|c|c|}
\hline 算法 & 场景 1 & 场景 2 & 场景 3 & 平均 \\
\hline 本文 & 0 & 0 & 0 & 0 \\
\hline 简单背景差分 ${ }^{[17]}$ & 0 & 0 & 0 & 0 \\
\hline 动态 ITTI 检测 ${ }^{[18]}$ & 75.00 & 64.71 & 62.50 & 67.40 \\
\hline Canny 边缘检测 ${ }^{[19]}$ & 16.59 & 5.40 & 5.05 & 9.01 \\
\hline 数学形态学滤波 ${ }^{[20]}$ & 10.84 & 5.68 & 4.38 & 6.97 \\
\hline 区域生长检测 ${ }^{[21]}$ & 3.20 & 3.95 & 2.14 & 3.10 \\
\hline
\end{tabular}

法效果还有待进一步实验验证.

对于机场跑道异物检测任务而言，漏检率(即 查全率)相较于错检率(即查准率)的影响与意义更 为重要, 若由算法检出的异物出现检测错误, 可由 后期人工或程序进行进一步的篮选，提高准确率; 但若算法未检测到地面异物, 出现漏检情况, 则需 要后期耗费大量人力和物力进行再次全面复查, 其对于异物检测任务的影响更为严重.

\section{4 结 语}

为解决机场跑道异物自主检测过程中存在的 检测结果环境鲁棒性低、小目标检测成功率低及漏 检率高的问题，本文提出了一种基于背景差分的 机场跑道异物分块检测与跟踪算法. 利用图像分 块后对 ORB 特征点的自适应加权提取, 将背景库 与待检测图像序列最相关帧进行对齐, 通过背景 差分获取异物检测结果，并对其进行多目标 KCF 跟踪; 在跟踪过程中，采用各子块局部跟踪算法降 低运算时间复杂度. 在多组不同场景下的实验结 果表明，与已有的基于图像的机场异物检测算法 相比，本文算法在异物整体错检率方面降低 $70 \%$ 以上，同时异物尺寸大于 $1 \mathrm{~cm} \times 1 \mathrm{~cm}$ 的情况下，整 体漏检率下降至 0 , 获得了较好的效果.

\section{参考文献(References):}

[1] FAA. AC 150/5220-24. Airport foreign object debris detection equipment $[\mathrm{S}]$

[2] Elrayes A, Ali M H, Zakaria A, et al. Smart airport foreign object debris detection rover using LiDAR technology[J]. Internet of Things, 2019, 5: 1-11

[3] Xu H Y, Han Z Q, Feng S L, et al. Foreign object debris material recognition based on convolutional neural networks[J]. EURASIP Journal on Image and Video Processing, 2018, 2018(1): Article No.21

[4] Mehdi G, Miao J G. Millimeter wave FMCW radar for foreign object debris (FOD) detection at airport runways[C] //Proceedings of 2012 9th International Bhurban Conference on Applied Sciences \& Technology. Los Alamitos: IEEE Com- puter Society Press, 2012: 407-412

[5] Nsengiyumva F, Migliaccio C, Brochier L, et al. 90 GHz, 3-D scattered field measurements for investigation of foreign object debris[J]. IEEE Transactions on Antennas and Propagation, 2019, 67(9): 6217-6222

[6] Zhan Lingming, Li Cuiyun, Ji Hongbing. Dynamic programming track-before-detect algorithm based on saliency map for infrared dim and small target[J] Journal of Computer-Aided Design \& Computer Graphics, 2019, 31(7): 1061-1066(in Chinese)

(詹令明, 李翠芸, 姬红兵. 基于显著图的红外弱小目标动 态规划检测前跟踪算法 [J]. 计算机辅助设计与图形学学报, 2019, 31(7): 1061-1066)

[7] Yu Zhijing, Tao Yongkui, Zheng Jianwen, et al. Detection algorithm for foreign object debris based on wavelet transform and morphology[J]. Science Technology and Engineering, 2020, 20(21): 8690-8695(in Chinese)

(于之靖, 陶永奎, 郑建文, 等. 基于小波变换与形态学的机 场跑道异物检测算法 [J]. 科学技术与工程，2020，20(21): 8690-8695)

[8] Zou Danhong. The application research of airport runway FOD detection algorithm based on difference image[D] Chengdu: University of Electronic Science and Technology. School of Information and Communication Engineering, 2014(in Chinese) (邹单红. 基于差分图像的机场跑道异物检测算法的应用研 究[D]. 成都: 电子科技大学信息与通信工程学院, 2014)

[9] Wang Jin. A hybrid airport runway's foreign object debris detection system[J] Science \& Technology Vision, 2019(9): 16-19(in Chinese)

(王瑾. 一种混合制式机场跑道异物探测系统[J]. 科技视界, 2019(9): 16-19)

[10] Dang Guolong, Sun Jin, Yang Liutao, et al. Foreign object debris detection based on improved visual attention mechanism[J] Aeronautical Computing Technique, 2018, 48(3): 103-106(in Chinese)

(党国龙, 孙瑾, 杨刘涛, 等. 基于改进视觉注意机制的跑道 异物检测算法[J]. 航空计算技术, 2018, 48(3): 103-106)

[11] Zou Zixiu. An improved moving target tracking algorithm based on MeanShift[J]. Journal of Jiujiang University: Natural Science Edition, 2020, 35(1): 45-47(in Chinese) (邹梓秀. 一种基于 MeanShift 改进的移动目标跟踪算法 $[\mathrm{J}]$. 九江学院学报: 自然科学版, 2020, 35(1): 45-47)

[12] Bewley A, Ge Z Y, Ott L, et al. Simple online and realtime tracking[C] //Proceedings of the IEEE International Conference on Image Processing. Los Alamitos: IEEE Computer Society Press, 2016: 3464-3468

[13] Wojke N, Bewley A, Paulus D. Simple online and realtime tracking with a deep association metric[C] //Proceedings of the IEEE International Conference on Image Processing. Los Alamitos: IEEE Computer Society Press, 2017: 3645-3649

[14] Henriques J F, Caseiro R, Martins P, et al. High-speed tracking with kernelized correlation filters[J]. IEEE Transactions on Pattern Analysis and Machine Intelligence, 2015, 37(3): 583-596

[15] Xiao Yiqing, Ge Hongwei. Adaptive correlation filtering algorithm suitable for long-term tracking[J]. Journal of Com- 
puter-Aided Design \& Computer Graphics, 2020, 32(1): 121-129(in Chinese)

(肖逸清, 葛洪伟. 适合长时跟踪的自适应相关滤波算法 [J]. 计算机辅助设计与图形学学报, 2020, 32(1): 121-129)

[16] Xiong Changzhen, Zhao Lulu, Guo Fenhong. Kernelized correlation filters tracking based on adaptive feature fusion[J] Journal of Computer-Aided Design \& Computer Graphics, 2017, 29(6): 1068-1074(in Chinese)

(熊昌镇, 赵璐璐，郭芬红. 自适应特征融合的核相关滤波 跟踪算法 $[\mathrm{J}]$. 计算机辅助设计与图形学学报, 2017, 29(6): 1068-1074)

[17] Li Dan, Zhang Geng, Shi Sen. Moving target detection method based on background subtraction and image sequence difference[J]. Telecommunications Science, 2017, 33(S1): 182-185(in Chinese)

(李丹, 张庚, 史森. 基于背景减除法和图像序列差分法的 运动目标检测 $[\mathrm{J}]$. 电信科学, 2017, 33(S1): 182-185)

[18] Dang Guolong. Research on the algorithm for detection and recognition of foreign object debris in airport runway[D]. Nanjing: Nanjing University of Aeronautics and Astronautics. Col- lege of Civil Aviation, 2018(in Chinese)

(党国龙. 机场跑道异物检测与识别的相关算法研究[D]. 南 京: 南京航空航天大学民航学院, 2018)

[19] Xu Hongke, Qin Yanyan, Chen Huiru. An improved algorithm for edge detection based on canny[J]. Infrared Technology, 2014, 36(3): 210-214(in Chinese)

(许宏科, 秦严严, 陈会茹. 一种基于改进 Canny 的边缘检测 算法[J]. 红外技术, 2014, 36(3): 210-214)

[20] Yue Bing. Research and implementation of detection and tracking moving object in image sequence based on OpenCV[D]. Hefei. University of Anhui. School of Electrical Engineering and Automation, 2016 (in Chinese)

(岳兵. 基于 OpenCV 的目标检测与跟踪算法的研究与实现 [D]. 合肥: 安徽大学电气工程与自动化学院, 2016)

[21] Xu Jing, Zhang He, Wang Xiaofeng. Target image segmentation method based on feature selection and region growing[J]. Journal of Detection \& Control, 2012, 34(1): 6-9+14(in Chinese)

(许敬, 张合, 王晓锋. 基于特征点和区域生长的目标图像 分割方法[J]. 探测与控制学报, 2012, 34(1): 6-9+14) 